November 2019

\title{
The Psychophysiology of Political Ideology: Replications, Reanalysis and Recommendations
}

\author{
Mathias Osmundsen1 \\ David J. Hendry2 \\ Lasse Laustsen 3 \\ Kevin B. Smith4 \\ Michael Bang Petersen5*
}

Forthcoming in Journal of Politics.

Please refer to published version for citation.

Short title: Psychophysiology of Political Ideology

Contact information:

1 Department of Political Science, Aarhus University, DK-8000, Aarhus C, Denmark, m.osmundsen@ps.au.dk

2 Department of Methodology, London School of Economics and Political Science, London WC2A 2AE, United Kingdom, d.hendry@lse.ac.uk

3 Department of Political Science, Aarhus University, DK-8000, Aarhus C, Denmark, 11@ps.au.dk

4 Department of Political Science, University of Nebraska, Lincoln, NE 68588-0328, United States, ksmith1@unl.edu

5 Department of Political Science, Aarhus University, DK-8000, Aarhus C, Denmark, michael@ps.au.dk

* Corresponding author 
Abstract: This article presents a large-scale, empirical evaluation of the psychophysiological correlates of political ideology and, in particular, the claim that conservatives react with higher levels of electrodermal activity to threatening stimuli than liberals. We (1) conduct two large replications of this claim, using locally representative samples of Danes and Americans; (2) re-analyze all published studies and evaluate their reliability and validity; and (3) test several features to enhance the validity of psychophysiological measures and offer a number of recommendations. Overall, we find little empirical support for the claim. This is caused by significant reliability and validity problems related to measuring threat-sensitivity using electrodermal activity. When assessed reliably, electrodermal activity in the replications and published studies captures individual differences in the physiological changes associated with attention shifts, which are unrelated to ideology. In contrast to psychophysiological reactions, self-reported emotional reactions to threatening stimuli are reliably associated with ideology.

Key words: Political ideology; threat-sensitivity; electrodermal activity; replication; measurement

Online appendix: Supplementary material for this article is available in the appendix in the online edition.

Data and replication materials: Replication files are available in the JOP Data Archive on Dataverse (http://thedata.harvard.edu/dvn/dv/jop).

Human-participants statements: This research involves human participants and was conducted in compliance with relevant laws. It was approved by an appropriate institutional ethics committee and was conducted in line with the ethical standards contained in the 1964 Declaration of Helsinki and its alter amendments.

Financial support: Support for this research was provided by the Velux Foundation grant VELUX33267 to Michael Bang Petersen. 
Going back to the 1950s, studies in both political science and political psychology have proposed that individual differences in political ideology do not just reflect differences in narrow political considerations but, rather, express broader sets of individual differences pertaining to personality, basic values or broader social outlooks (Hibbing et al., 2014; Jost, 2006). In particular, a common argument has been that a conservative political ideology is likely to be endorsed by individuals motivated to reduce threats in their daily lives. In this view, so-called "threat-sensitive" individuals find the order inherent in a conservative ideology attractive. A large range of studies support this basic assertion using diverse methods, including assessing differences between liberals and conservatives in selfreported need to reduce insecurity (e.g., Jost et al., 2009), observing the living spaces of liberals and conservatives (e.g., Carney et al., 2008), assessing personality differences between liberals and conservatives (e.g., Gerber et al., 2010) and investigating the impact of threatening events (e.g., terrorist attacks) on public endorsement of conservative policies (e.g., Merolla \& Zechmeister, 2009).

Recently, the literature on the broader underpinnings of ideological differences has turned towards their potential biological roots. A consistent finding across studies using methods from behavioral genetics (primarily, but not exclusively, twin studies) is that individual differences in political ideology are genetically heritable (Hatemi \& McDermott, 2012). Furthermore, psychologists have used techniques from neuroscience (such as fMRI) to identify neural differences between liberals and conservatives that correspond to differences in threat-sensitivity, especially relating to the structure and function of the amygdala, a brain region involved in the processing of fearful, threatening and otherwise emotionally vivid stimuli (for a review, see Jost \& Amodio, 2012).

While psychologists have turned towards neuroscience in understanding the biological underpinnings of political ideology, political scientists have turned towards techniques from psychophysiology. Whereas measures obtained via neuroscience methods are expensive and require extensive training, psychophysiological studies are far less costly to conduct (Soroka, 2019). In particular, this work has focused on the measure of skin conductance or electrodermal activity (EDA), 
an index of sympathetic nervous system arousal obtained by measuring microscopic changes in sweatproduction via electrodes on the fingertips (Figner \& Murphy, 2011). The seminal finding was established by Oxley et al. (2008) who found that conservatives responded with higher EDA than liberals when viewing images of diverse threats such as spiders, maggots and guns. Since then, a number of follow-up articles have been published, all using psychophysiology to shed light on the psychological underpinnings of political ideology (Dodd et al., 2012; Knoll et al., 2015; Smith et al. 2011).

The aim of this article is to establish the first large-scale, empirical evaluation of the literature on the psychophysiological correlates of political ideology and, in particular, of the claim that relative to liberals, conservatives react with higher levels of EDA responses to threatening visual stimuli. We evaluate the evidence in favor or against this claim by (1) conducting a large replication effort, fielding laboratory experiments based on locally representative samples of Danes and Americans with a combined sample size of 348 , over seven times the number of participants in the original Oxley et al. (2008) study; (2) re-analyzing all published studies with the specific aim of establishing their reliability and validity; and (3) examining several coding features to enhance the validity of the utilized measures and, on this basis, offering a number of recommendations.

Now, a little more than a decade after the psychophysiological study of political ideology was initiated, we believe it is of critical importance to evaluate the introduction of psychophysiological methods into political science. First of all, claims based on these methods have been viewed as controversial within recent political science research, sparking debates on, e.g., the changeability of political views (for an overview, see Hibbing, 2013). Second, the evidence from the existing studies is mixed, with several studies observing supportive evidence and one study failing to replicate the finding (Knoll et al., 2015).1 Relatedly, psychophysiological measures are shaped by "a large number of

1 In the process of revising this article, a preprint of another large-scale replication effort became available. Bakker et al. (2019) field two conceptual replications as well a pre-registered direct replication of Oxley et al. (2008). All of these 
nuisance variables" (Tomarken, 1995: 390), but little has been done to evaluate the consequences of the properties of psychophysiological measures in political science. This is particularly noteworthy because a lack of attention to measurement properties is also present in the psychophysiological field itself (Ogorevc et al., 2013; Tomarken, 1995) and because there are many ways to collect and code psychophysiological measures (Murphy \& Figner, 2012). Third, because psychophysiological methods are cheap and appear easy to administer, there has recently been a proliferation of interest in using these methods, also outside the study of political ideology. Studies on issue attitudes (Aarøe et al., 2017), political communication effects (Coe et al., 2017) and party cue effects (Petersen et al., 2015) all rely on measures of electrodermal activity. From the larger field of political behavior research, it is of key importance to pause and ask: How well do these measures capture the constructs we are interested in?

Our main contribution is to raise significant methodological concerns about the use of psychophysiological methods in political science. We are only able to replicate the original Oxley et al. (2008) finding in the United States, not in Denmark. Furthermore, our re-analyses show that our replications and many past studies used measures that by conventional standards are unreliable (at least as measures of the target constructs) and that the available data, to a larger extent than previously recognized, does not support the existence of an association between physiological threat-sensitivity and political ideology. Still, our recommendations point a way forward for the use of psychophysiological measures in political science. We explore several possible protocols for addressing issues related to reliability and measurement validity and we identify the coding decisions that will most likely yield reliable measures. However, as we discuss on the basis of the psychophysiological literature and additional analyses of the present data, these reliable measures do not capture individual differences in threat-sensitivity. Instead, they are better seen as capturing

efforts fail to replicate. We encourage readers to consult Bakker et al. (2019), which is aligned with and reinforces the conclusions of the present article. 
individual differences in the physiological activity associated with attention shifts. This suggests that measures of electrodermal activity could be better incorporated into political science research by being firmly anchored in theoretical frameworks that explicitly address how such individual differences are relevant to political attitudes and behavior.

\section{Methodological Concerns: Replicability, Reliability and Validity}

The aim of this article is to provide a methodologically thorough assessment of the evidence for an association between political conservatism and threat-sensitivity measured as individual variation in EDA when processing images with negative (e.g., threatening) content. We examine this association using four central criteria for scientific contributions: (1) the replicability of the association; (2) the reliability of EDA as a psychophysiological measure; (3) the measurement validity of both EDA and measures of political conservatism; and (4) the external validity of the association.

Replicability is a hallmark of science and the social sciences increasingly recognize the value of replication studies (Open Science Collaboration, 2015). Still, few have attempted to replicate the original Oxley et al. (2008) finding. Teams associated with the original author set have reported two successful follow-up studies on the association (Dodd et al., 2012; Smith. et al., 2011). However, while not disclosed in Dodd et al. (2012), the EDA analyses in that article is based on exactly the same dataset as Oxley et al. (2008) with slightly different operationalizations.2 Furthermore, an independent replication attempt failed to identify the association in a sample of American undergraduates (Knoll et al., 2015). While subsequent work has identified a number of key differences in the procedures

2 For example, Dodd et al. (2012) focus on EDA reactions to three "aversive" images ("a spider on a man's face", "an open wound with maggots in it", and "a crowd fighting with a man") and Oxley et al. (2008) focus on EDA reactions to three "threatening" images ("a very large spider on the face of a frightened person, a dazed individual with a bloody face, and an open wound with maggots in it"). The image of "a crowd fighting a man" is not mentioned in Oxley et al. (2008) and, hence, it is not discussed why this is not considered a "threatening" image. It should also be noted that Dodd et al. (2012) includes analyses of data beyond Oxley et al. (2008), including analyses of physiological reactions to political images and a separate study using eye-tracking. 
between the original study and the independent replication (Peterson et al., 2016), the lack of replicability from independent labs raises concerns. In this regard, it is important to note that a number of studies have recently been published with relevant psychophysiological data (Aarøe et al., 2017; Coe et al., 2017; Petersen et al., 2015). While these studies focus on different research questions, they all include measures of both political ideology and EDA measures of threat-sensitivity, although they do not report the associations between the two variables. Consequently, there exists a pool of additional data that can be used to examine the replicability of the association between physiological responses to threat and political ideology.

Another key criterion of research concerns the reliability of the measures used. As argued by Tomarken (1995: 389), "psychophysiological measures are only useful to the degree that they meet the same psychometric criteria that are commonly invoked for self-report and behavioral measures." In this regard, it is relevant that EDA is influenced by a range of factors that are likely to vary randomly and arbitrarily across individuals (Figner \& Murphy, 2011; Tomarken, 1996). These factors include outside noises, deep breaths, coughs, room temperature, bodily movements, thickness of the skin of the fingertips, pre-experiment arousal (e.g., from having biked to the lab) and so forth. Yet, there has been a lack of attention to the measurement properties of EDA measures. In an assessment of the broader psychophysiological literature, Tomarken (1995: 389) concludes that "despite its evident importance, the reliability of psychophysiological measures recorded on a single occasion is rarely assessed or reported." In a similar assessment, Manuck et al. (1989: 368) note that "few investigators have examined the reproducibility of psychophysiological responses over multiple experimental sessions". These assessments were echoed as recently as in 2013: "Almost all papers discussing skin conductance measurements describe the measurement results in absolute terms using an appropriate measurement unit (...), but accuracy and consequently reliability of reported measurement results is seldom questioned and investigated" (Ogorevc et al, 2013: 2994). These remarks certainly fit the studies using psychophysiology within political science. For example, published studies rarely report 
standard tests of reliability such as Cronbach's alpha. Thus, we simply do not know if measures of EDA in political science research are empirically reliable.

In addition to concerns about the reliability of the measures used in past studies, it is also relevant to note concerns about their measurement validity. First, as noted above, EDA was originally validated as a measure of physiological arousal. By specifically examining EDA responses to negative visual stimuli, studies in political science have sought to obtain discrete measures of threat-sensitivity. At the same time, these studies lack regular validity tests such as tests of convergent validity (i.e., do EDA responses to different threatening images converge?) and discriminant validity (i.e., do EDA responses to threatening images differ from EDA responses to, say, positive images?). These are particularly relevant questions as some researchers have recently argued that physiological differences between liberals and conservatives relate less to threat and more to individual differences in general arousal, with conservatives being more easily aroused than liberals (Tritt et al., 2014). Second, there is ambiguity about the nature of the stimuli used to measure physiological threat-sensitivity. In the original study by Oxley et al. (2008), the negative stimuli included both images of threats to physical safety and images that provoked disgust. In a subsequent paper by Smith et al. (2011), the negative stimuli exclusively focused on disgust. Other physiological studies in political science have also used disgust- and threat-related stimuli to varying extents. There are strong theoretical reasons and ample empirical support for an association between self-reported measures of threat-sensitivity and disgustsensitivity, on the one hand, and political ideology, on the other hand (Terrizzi et al., 2013). But at present no studies have directly compared and discriminated between these two forms of stimuli in the context of psychophysiology.

A question about measurement validity can also be raised regarding measures of ideology and which ideological dimensions are associated with threat-sensitivity. Oxley et al. (2008) used items from a Wilson-Patterson political attitude scale as their ideological measure and found that higher threat-sensitivity was associated specifically with preferences for "socially protective policies." 
Yet, ideological differences extend beyond the domain of social attitudes: Attitudes in the economic domain (e.g., relating to government redistribution) are also important. At present, however, we do not know whether physiological measures of threat-sensitivity are only associated with the social components of ideology, as prior ideological measures have not included economic components. This question is made salient by the fact that in the broader literature on the psychology of ideology there is a debate about whether the social and economic components of ideology constitute a single liberalconservative dimension or two separate dimensions (e.g., Jost et al., 2009; Malka et al., 2017).

Finally, existing studies raise concerns about the external validity of the association between threat-sensitivity as measured by EDA and ideology. Existing studies have all been conducted in the United States with non-representative samples. As emphasized by Hibbing et al. (2014: 303), "[a]dditional studies are needed [...] because much of the extant physiological work is based on small, geographically constrained samples and much of the psychological work relies on college undergraduates who may have yet to form stable political attitudes." Even if the original findings hold, we remain ignorant of whether the association between physiological markers of threat-sensitivity and ideological orientations generalizes to populations outside the United States. Looking to the broader literature on the psychology of ideology, two contrasting expectations emerge. On the one hand, it is possible that support for socially protective policies is the universal output of psychological mechanisms for threat-management across countries and cultures (see, e.g., Aarøe et al., 2017). On the other hand, some studies suggest that there is contextual variation in the link between ideology and psychological measures related to threat-sensitivity such as feelings of uncertainty (Malka et al., 2017). One explanation for this is that the output of threat-management mechanisms is not support for particular kinds of policies but rather support for the status quo (often referred to as system justification; Jost et al., 2004). In this case, physiological measures of threat-sensitivity would be associated with conservatism in conservative countries and contexts, but with liberalism in liberal 
countries and contexts. In order to examine this, we need cross-national replications in countries that differ in the ideological profile of their political systems.

\section{Replications: Cross-National Laboratory Studies}

To provide an initial examination of the issues of replicability, reliability, measurement validity and external validity, we conducted two cross-national and well-powered conceptual replication studies of Oxley et al. (2008).

\section{Sampling}

We executed parallel laboratory studies in Aarhus, a midsized university town in Denmark, and in Lincoln, a midsized university town in the Midwest of the United States. The site of the US study is the same as in Oxley et al. (2008) and the Danish sample provides leverage in establishing external validity. Thus, Denmark provides a "liberal" political context, both in terms of economic and social issues, with a large, universalistic welfare state and liberal policies and public opinion regarding, for example, abortion and the rights of homosexuals.

In Denmark, the construction of the samples and the invitations to participate were carried out by the YouGov survey agency from September to November, 2015; in the United States, recruitment was carried out by an agency at the University of Nebraska from June to October, 2016. The Danish sample consisted of 178 participants, while 170 participants took part in the US study. The number of participants in each sample is three to four times higher than in the original Oxley et al. (2008) study.3 Thus, our much larger sample sizes allow us to zoom in on the ideological extremes after, rather than before, collecting data. The composition of the samples was chosen to be

\footnotetext{
3 One difference between the sampling strategy of our replication studies and the original study, however, was that Oxley et al. (2008) specifically sampled individuals with "strong political convictions." To assess whether this difference in sampling strategy impacts the findings from the replications, Online Appendix Section 5C, reproduces the present analysis while removing participants with weak political convictions. This does not change the conclusions reported here.
} 
representative of the broader populations of the two cities with respect to gender, age and education. In the Danish sample, $52 \%$ of participants were female, the average age was 43 years $(\mathrm{SD}=15$, Min $=18, \operatorname{Max}=70), 9 \%$ of the participants had no high school diploma, $41 \%$ were high school graduates or similar, $6 \%$, had less than two years of college, $27 \%$ had 3-4 years of college, and $18 \%$ had more than 4 years of college. The median household income was $\$ 55,000$ to $\$ 64,999$. In the US sample, $58 \%$ were female; the average age was 50 years $(\mathrm{SD}=17, \operatorname{Min}=20$, Max $=85) ; 9 \%$ had no high school diploma, $25 \%$ were high school graduates or similar, $13 \%$ had less than two years of college, $31 \%$ had 3-4 years of college, and $21 \%$ had more than 4 years of college; and the median household income was between $\$ 55,000$ and $\$ 64,999$.

\section{Measures}

In both the Danish and American laboratory studies, we recorded participants' EDA while they viewed a series of images on a computer screen. While our images were not identical to the images in Oxley et al. (2008), we chose them in close consultancy with authors of that study. The participants viewed 24 images in random order, where each stimulus image was shown once for eight seconds, and was preceded by an interstimulus interval (ISI), a blank screen lasting six seconds. To allow us to examine issues of measurement validity, we chose images that tapped into four distinct emotions. Six images elicited feelings of threat (e.g., a man with a knife, a man pointing a gun towards the screen), six elicited disgust (e.g., a man eating maggots, a baby with an open wound), six had positive emotional content (e.g., a waterfall, a couple kissing) and six were neutral (e.g., an umbrella, a dustpan).

To obtain our measure of EDA, we followed the "log-and-subtract" procedure from Oxley et al. (2008). Specifically, we first took the average logged EDA response during exposure to the stimulus image and subtracted from that the average logged EDA response from the preceding ISI. This procedure allows us to isolate the EDA response to a specific image corrected for between-subject 
baseline variations in EDA. 4 We then combined the changes in EDA for the six images within each of the four emotion categories to produce an overall mean EDA response within that image category (e.g., we created an overall measure of EDA response to the six threatening images). Below we assess the reliability and measurement validity of these measures. We removed one outlier in the American data with EDA responses 15 standard deviations above the mean EDA response.5

We used four measures of political ideology to address concerns about measurement validity. Following Oxley et al. (2008), the first measure was a Wilson Patterson 20-item policy issue battery $(\alpha \mathrm{DK}=.82 ; \alpha \mathrm{US}=.84)$. To assess the potential distinction between social and economic components of ideology, our second and third measures were a 5-item Social Conservatism Scale ( $\alpha \mathrm{DK}$ $=.82 ; \alpha \mathrm{US}=.79)$ and a 6-item Economic Conservatism Scale $(\alpha \mathrm{DK}=.82 ; \alpha \mathrm{US}=.85)$, taken from Slothuus et al. (2010).6 Our final measure was a single-item Ideological Self-Placement, where participants placed themselves from "Most liberal" ("Extremely left-wing" in the Danish sample) to "Most conservative" ("Extremely right-wing” in Denmark; see Online Appendix Section 2B for detailed descriptions). Higher values on all scales indicate greater conservatism.

In all models, we control for gender, age, educational level, and income (cf. Oxley et al., 2008). Gender is a binary indicator for female (female $=1$, else $=0$ ), while age (measured in years) and education level and income (measured on ordinal scales) are standardized to have a mean of 0 and standard deviation of 1.

\footnotetext{
4 We also explore a novel alternative correction strategy: Using reactions to the neutral images as baseline. We report these analyses in Online Appendix 5E. The findings using this alternative method are in line with the results presented in the main text.

5 We also obtained measures of electromyography over the corrugator supercilii muscle. This measure was not utilized in Oxley et al. (2008) and we report the findings for this measure in the Online Appendix Section 5B.

6 As these items originate in the Danish National Election survey, Online Appendix 5F provides a replication using another measure of social conservatism - Right-Wing Authoritarianism - and a different economic conservatism measure - Social Dominance Orientation - both of which may fit better in a US context. Online Appendix 5F also includes the Society Works Best scale, which constitutes another measure of political ideology.
} 


\section{Assessing Reliability and Validity of the EDA Measures}

We first address concerns about the reliability and validity of physiological measures of threatsensitivity, focusing on participants' EDA responses to the threatening and disgusting images. We examine reliability in two ways. First, we calculate Cronbach's $\alpha$ for EDA responses to the negative images (i.e., threatening and disgusting images). If our physiological measures are reliable, Cronbach's $\alpha$ should be high. Second, we investigate whether the study design provides a strong measurement signal. As argued above, prior studies isolate the EDA signal by taking the difference between EDA responses to threatening images (EDAstimulus) and EDA responses when viewing the preceding black screen (EDAInterstimulus). As a measure of reliability, we thus examine the correlations between EDAstimulus and EDAInterstimulus responses to the threatening and disgusting images, respectively. If exposure to these images produce a strong signal, EDAstimulus should be much larger than EDAInterstimulus, and thus they should not correlate highly. In contrast, the signal is weak if EDAstimulus and EDAInterstimulus are almost identical. If a measurement tool is precise and measured without noise, a weak signal would not necessarily constitute a problem. Yet, because EDA is influenced by multiple confounding factors, a weak signal would be a cause of concern here.7

To identify the measurement validity of our EDA measures, we first examine convergent validity by testing whether participants have similar EDA responses to different threatening images. If EDA responses to diverse sets of negative images reflect the same latent trait, they should correlate positively. To next examine the measures' discriminant validity, we test whether EDA responses to threatening images correlate with EDA responses to other image types (e.g., positive and neutral

\footnotetext{
7 In their discussion of the use of psychophysiology as a measure of individual differences, Manuck et al. (1989: 367) write: "Because correlations of baseline measurements with both task values and arithmetic change scores are rarely, if ever, perfect, individuals' physiologic states during periods of stimulus presentation exhibit residual variability that cannot be accounted for by a knowledge of baseline values alone. It is this residual variability that might best be considered as capturing variability as indicative of the psychophysiologic "reactivity" of individuals." However, in contrast to the present data, the example referred to in this discussion exhibited a correlation of only $r=.55$ between physiological reactions during the inter-stimulus interval and the stimulus interval.
} 
images). If EDA responses to different image types reflect distinct latent traits, the correlations across image categories should be low.

Turning first to our reliability tests, we find that our measures are very unreliable. Cronbach's $\alpha$ for EDA responses to threatening images are low in both our samples. (Denmark: $\alpha$ Threat Images $=.11$; United States: $\alpha$ Threat Images $=.14)$; indeed, EDA responses to disgusting images were so unreliable that we could not calculate scale reliability coefficients. Further, participants' EDAstimulus responses to both threatening and disgusting images correlated extremely highly with their EDAInterstimulus responses ( $r$ values $>.97$ in both Denmark and the United States). The high correlation between EDAstimulus and EDAInterstimulus implies that both measures reliably capture the same common quantity. As we return to in the conclusion, this common quantity most likely reflects individual differences in baseline physiological reactivity. But the high correlation also implies that the real quantity of interest - EDA responses to threatening images - is at best only very weakly captured. The combination of a very high correlation and numerous potential confounds for EDA responses suggests that the tiny difference between EDAstimulus and EDAInterstimulus could reflect noise rather than a signal of EDA reactivity to threat. This interpretation is bolstered by another result: Difference-of-means tests reveal that participants do not have stronger EDA responses for negative images than during the interstimulus intervals. 8 If each of EDAstimulus and EDAInterstimulus largely reflects some common quantity plus an error term, the computed difference score is essentially just random error.

Our measure of convergent validity also performs poorly. The average inter-item correlations between the six threatening images and between the six disgusting images are very low in both countries, suggesting that they do not reflect the same underlying traits (Denmark: rThreat Images $=$

8 In both Denmark and the United States, we find no statistically significant difference between EDA responses for threatening images versus EDA responses for preceding interstimulus interval (Denmark: $t=-1.11, p=.27$; United States: $\mathrm{t}=-1.44, \mathrm{p}=.15$ ). In Denmark, participants had stronger EDA responses during the interstimulus interval than during exposure to disgusting images $(\mathrm{t}=-3.53, \mathrm{p}<.001)$; the difference was insignificant in the United States $(\mathrm{t}=-.94$, $\mathrm{p}=.35)$ 
.02 ; rDisgust Images $=.10$; United States: rThreat Images $=.03$; rDisgust Images $=.06$ ). Given the unreliable measures and low convergent validity, it is unsurprising that our final divergent validity test shows that EDA responses to threatening and disgusting images do not correlate with EDA responses to positive and neutral images. In the United States, the correlations between image categories varied between $r=-.16$ and $r=.14$ and, in Denmark, they varied between $r=-.15$ and $r=.08$. In most circumstances, this would suggest that divergent validity is high but, given the other measurement issues, it is difficult to interpret these correlations.

We return to a thorough discussion of these signs of extreme unreliability in subsequent sections.9 For now, we set aside the measurement problems and instead test whether we are able to replicate the main finding in Oxley et al. (2008) that EDA responses to threatening images correlate positively with political conservatism.

\section{Results}

We present the results in Figure 1, which displays estimated regression coefficients from models where we regressed our four measures of political ideology on EDA responses to threatening, disgusting, neutral and positive images.10 We estimated models for EDA responses to each of the four image categories separately, and we estimated the models for Denmark and the United States separately, as well as for the combined sample. Each model included the control variables discussed above, and in the combined sample, we additionally controlled for country. To compare the sizes of the estimated coefficients, we standardized all the measures. Horizontal bands display $95 \%$ confidence intervals.

\footnotetext{
9 It is relevant to note that all processing of the raw data was initially conducted in one laboratory. To ensure the reliability and measurement validity problems did not reflect miscodings, the other laboratory visually inspected and subsequently re-processed and re-coded all raw data. We did note some differences and report analyses of the re-processed data in Online Appendix Section 5D. Taken together, however, the re-processing of the data did not substantially change the results. 10 In Online Appendix section 5G, we estimate the relationship between EDA responses and political ideology with random effects models where we treat participants' reactions to each image as the unit of analysis. The results do not differ appreciably from those presented here. Future studies might increase the reliability of estimates by exposing participants to many more images and then use this multi-level approach.
} 
For the United States, the results largely support the original Oxley et al. (2008) study: Individuals displaying higher EDA responses to threatening images were more likely to support conservative policies on the Wilson-Patterson Battery $(b=.33, \mathrm{p}=.013)$ and the Social Conservatism Scale $(b=.33, p=.001)$. On the other hand, EDA responses to threat did not correlate significantly with the two other ideological measures in the US sample (Economic Conservatism: $\mathrm{b}=.12, \mathrm{p}=.280$; Left-Right self-placement: $\mathrm{b}=.04, \mathrm{p}=.181)$. While this might indicate a difference in associations between threat-sensitivity, on the one hand, and social and economic conservativism, on the other, further analyses show that this difference is not in itself statistically significant $(p=.118)$. In the US, reactions to disgusting images were also generally associated with conservatism, although the associations are appreciably weaker and not significant at conventional levels. We did not detect any systematic relationship between EDA responses to neutral and positive images on the one hand, and political ideology on the other.

Results differed markedly among the Danish participants. In general, we did not detect a systematic relationship between EDA responses to any of the four image types and political ideology. If anything, stronger EDA responses to threatening images were associated with more liberal preferences, although not significantly so for any of our measures. Further, examining the interaction between EDA responses and country, we found that the relationships between EDA responses to threat and the Wilson-Patterson Battery and the Social Conservatism Scale were statistically different from one another in the two countries (pwilson-Patterson $=.007$; psocial Conservatism $=.003$ ). Because of these country differences in the direction of the relationship between EDA responses and political ideology, none of the combined results for Denmark and the United States were statistically significant at conventional levels. 
Figure 1. Associations between four measures of political ideology and EDA responses to threatening, disgusting, positive and neutral images among Danes, Americans and the combined samples.
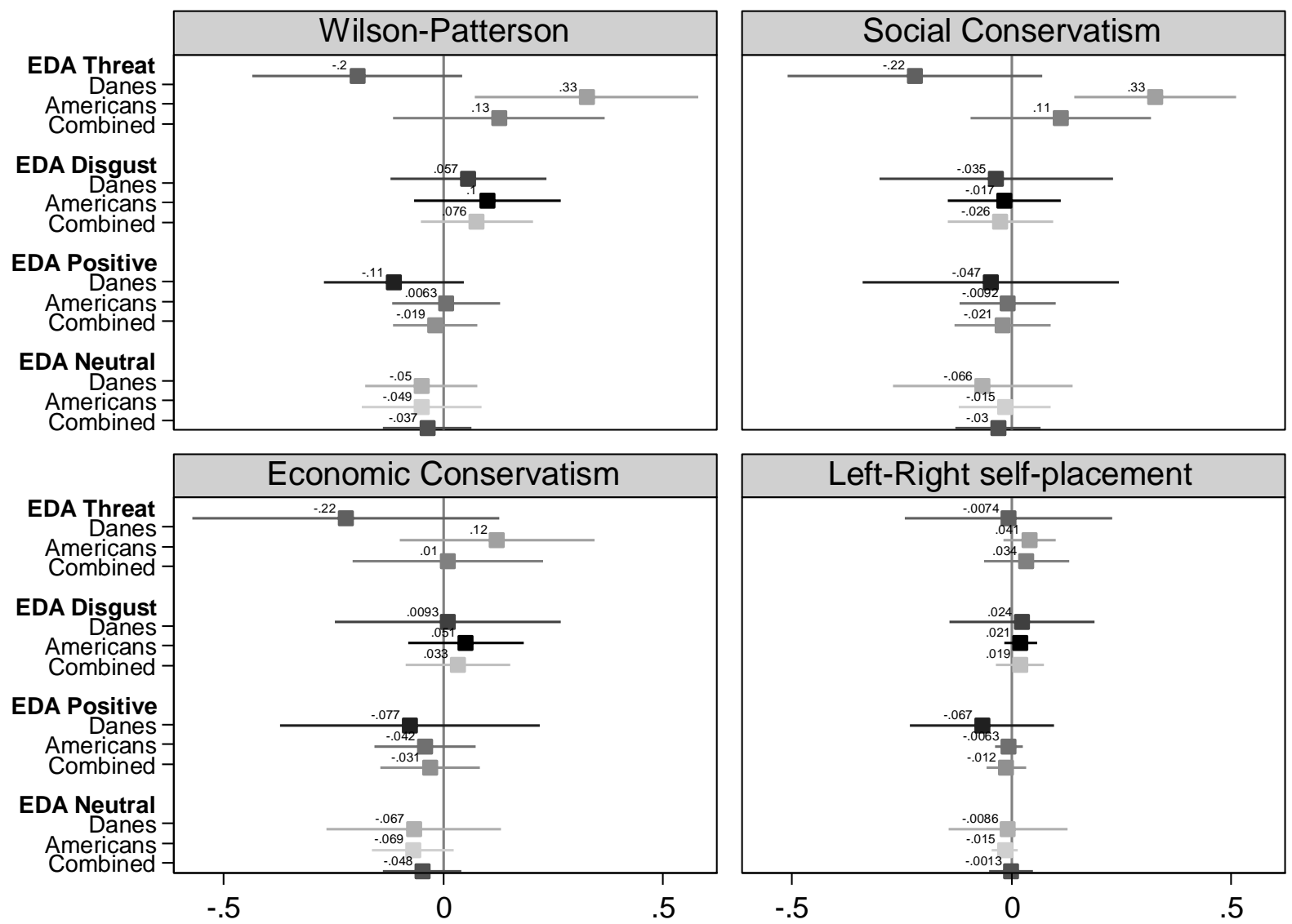

Notes $\mathrm{N}=155$ (DK) / 163 (US) / 318 (Combined). Entries are coefficient estimates from OLS regression analyses of the associations between ideology measures and electrodermal activity when viewing threatening, disgusting, positive and neutral images. We estimated models for EDA responses to each of the four image categories separately, and we estimated the models for Denmark and the United States separately, as well as for the combined sample. We included a binary indicator for gender, and age, education level, and household income standardized to have a mean of 0 and a standard deviation of 1 . See Online Appendix 2 for details.

\section{Assessing the Association with More Reliable Measures}

Past studies have supported the existence of an association between threat-sensitivity and political conservatism using other, often self-reported, measures. At the same time, there is a debate about whether this association depends on the ideological context. To assess whether the lack of an association in the Danish replication study reflects a true effect or a false-negative due to measurement 
error, we examine an additional measure that we collected in the replication studies: self-reported emotional reactions to the images.

We note that it may seem curious to some readers that we validate psychophysiological results using self-reported measures, given that psychophysiological measures are explicitly employed to move beyond self-reports. We are not the first to do so: Key validations of psychophysiology as a measure of arousal are themselves based on self-reports (see Lang et al., 1993).

\section{Materials and Methods}

In the Danish and American laboratory samples, we asked participants in our two samples to rate their self-reported emotional reactions to a subset of the 24 images previously shown on two dimensionsvalence and arousal. Specifically, we presented participants with two images from each of the four categories: two threatening images (a man with a knife, a snake), two positive images (a skydiver, a romantic couple kissing), two disgusting images (a baby with a tumor, worms) and two neutral images (a plate, a mug). For each image, we measured valence by asking on 9-point scales if participants responded with "Happy, positive feelings" or "Unhappy, negative feelings" when viewing that image. We measured arousal by asking on 9-point scales if participants had "No reaction" or a "Strong reaction" when viewing the image. We then constructed indices for each of the four image categories by combining responses to the two images from that image category; scaled so higher values indicated more negative reactions and higher arousal, respectively. As outlined in Online Appendix Section 2C, all indices show satisfactory levels of reliability. Section $2 \mathrm{C}$ also shows that the self-reported ratings do not generally correlate with physiological reactions to those same images. The only exception is the correlation between self-reported valence ratings and physiological reactions to threatening images, where correlations are significant and positive: rUnited States $=.16$; rDenmark $=.31$. 
In the analyses that follow, we relied on the same four measures of political ideology as our dependent variables: Wilson-Patterson, Social Conservatism, Economic Conservatism and LeftRight self-placement. Again, we use measures scored with means of 0 and standard deviations of 1.

\section{Results}

Here we focus on the valence ratings, which directly measures sensitivity to the threatening nature of the stimuli (see Online Appendix 2E for similar analyses and results using the arousal ratings). We present the findings in Figure 2. As before, the figure displays estimated regression coefficients, but this time from models where we regress our four measures of political ideology on self-reported valence reactions to the four image categories. In the figure, positive coefficients indicate that negative evaluations of the images are associated with conservatism. We estimated the models separately for Denmark and the US as well as for the combined sample. In all models, we included the same set of covariates described above in the context of Figure 1.

In contrast to physiological responses to threat, self-reported reactions to the threatening images were associated in both Denmark and the United States with more conservative beliefs, but only significantly so for the two measures that arguably reflect social conservatism: the WilsonPatterson scale $(\mathrm{bDK}=.12, \mathrm{pDK}=.025 ; \mathrm{bus}=.24, \mathrm{pus}=.007)$ and the Social Conservatism scale $(\mathrm{bDK}$ $=.19, \mathrm{pDK}=.034 ; \mathrm{bus}=.17$, pus $=.006)$. In other words, participants who rated the threatening images as more negative were more socially conservative. In Denmark, the difference in strength of the association for the economic and socially conservative measures was statistically significant $(\mathrm{p}<.005)$ and, in the US, it was marginally significant $(\mathrm{p}=.11)$. Participants' reactions to the disgusting images were also associated with more conservative preferences, but not significantly so for any of the ideology measures. We did not detect a systematic relationship between participants' evaluations of the positive and neutral images, on the one hand, and their ideological orientations, on the other hand. 
In sum, these analyses support the argument that the failure to identify an association in the Danish data could be due to the measurement properties of the psychophysiological measures rather than a contextual difference between Denmark and United States. This underscores the need to validate findings that employ psychophysiology using measures with more desirable measurement properties.

Figure 2. Associations between four measures of political ideology and self-reported valence of responses to threatening, disgusting, positive and neutral images among Danes and Americans.
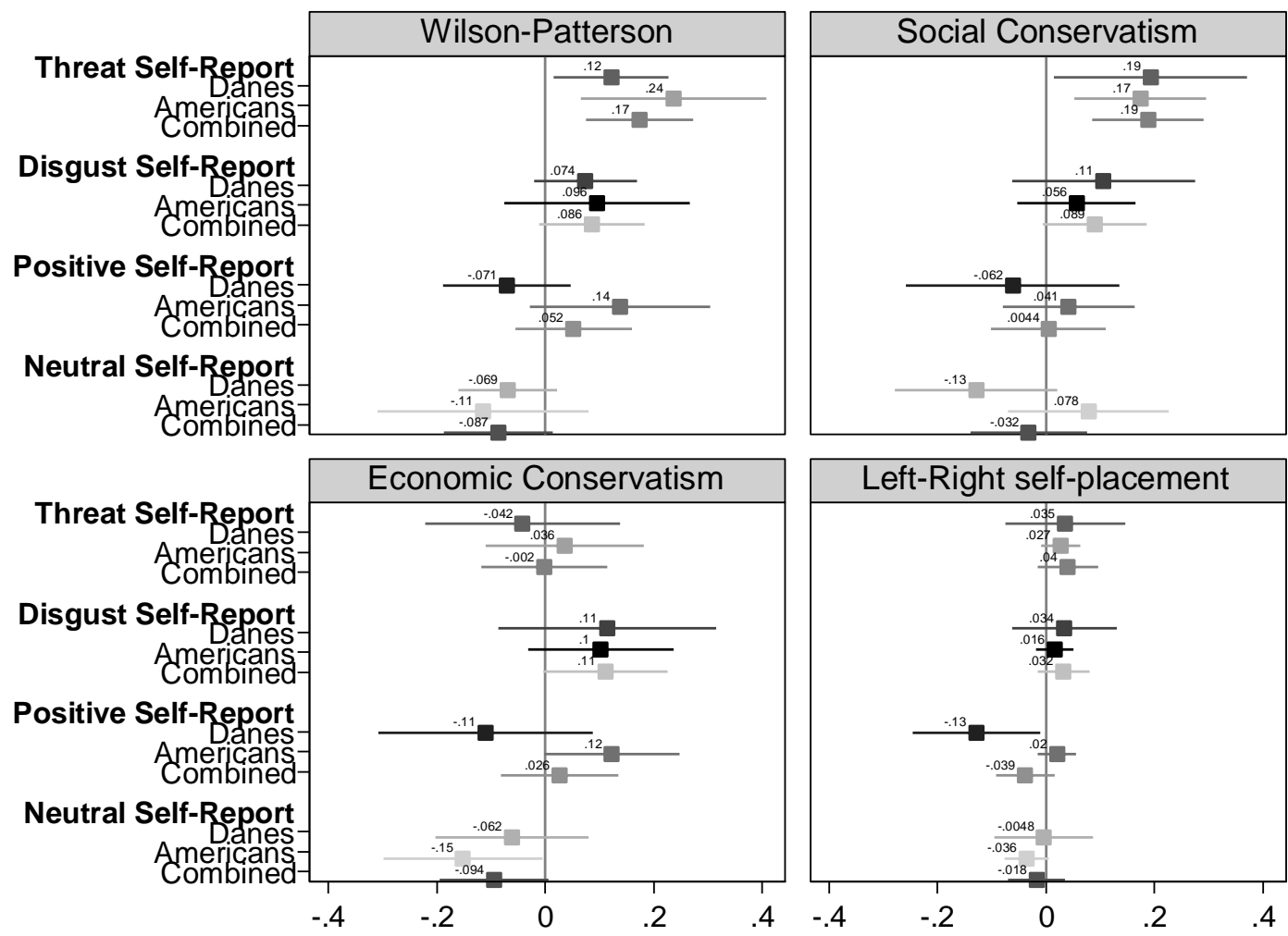

Notes. $\mathrm{N}=155$ (DK) / 163 (US) / 318 (Combined). Entries are coefficient estimates from OLS regression analyses of the associations between ideology measures and self-reported valence reactions when viewing threatening, disgusting, positive and neutral images. We estimated models for self-reported responses to each of the four image categories separately, and we estimated the models for Denmark and the United States separately, as well as for the combined sample. We included a binary indicator for gender, and age, education level, and household income standardized to have a mean of 0 and a standard deviation of 1 . See Online Appendix 2 for details. 


\section{Exploring Possible Methods for Increasing the Measurement Properties of Physiological}

\section{Reactions}

The findings from the self-reported test suggest that physiological reactions to threatening pictures ought to be associated with political conservatism in both Denmark and United States. Yet, to identify such an association, we need to counter-balance the documented issues in the measurement of threatsensitivity. Above, we noted the general lack of associations between self-reported ratings for images and physiological reactions to the images and, although associations were significant for threatening pictures, they were at the same time relatively low. This suggests that we cannot assume that all threatening images are equally threatening in general or for each individual respondent. In this section, we explore whether it is possible to take these issues into account by combining self-reported and physiological measures.

Specifically, the method we explore relies on ratings of each picture used in our replication studies on those specific dimensions that are key for differentiating from a measurement perspective (e.g., threat and arousal). We then analyze the data at the level of reactions to a specific picture as the unit of analysis. This allows us to model interactive effects between the properties of the image (as defined by the ratings) and physiological reactions to the image on measures of political ideology. This could increase, first, measurement validity as we no longer assume that all threatening pictures are equally threatening or that all non-threatening pictures are equally non-threatening. Instead, we directly obtain verifications of how threatening each picture is and therefore can test whether physiological reactions become more strongly associated with ideology with continuous increases in the degree of threat in the stimuli. Because the effect of the reactions is modelled separately for each image, this approach also does not rely on the assumption that the reactions emerge from a common latent trait. Second, this method could increase reliability as it expands the number of data points substantially. Thus, it is now possible to model the associations on the basis of reactions to many images rather than just a small handful of pictures (as is the case in both our replications and in existing 
studies). At the same time, it is worth mentioning that this modelling approach certainly does not solve all issues identified above. In particular, it cannot circumvent the fact that the measures of each reaction are themselves extremely noisy given the sizeable correlations between reactions to the image and the preceding ISI.

\section{Materials and Methods}

We examine whether self-reported ratings can enrich psychophysiological data in two different ways: first, we rely on the participants' own self-reported ratings of the eight images, which were also utilized in the previous test. In this analysis, we thus interact physiological reactivity to a picture with the participants' own ratings of the very same picture. This analysis allows us to test whether associations depend on whether participants' self-reported and physiological reactions to a specific image align or not. Second, we collected a dedicated rating survey where we had all images rated on relevant dimensions. In this analysis, we thus interact physiological reactivity to a picture with the average rating of the picture by external raters.

The rating survey was fielded as an approximately representative online survey with 450 participants, collected in Denmark in June 2018. In the survey, we asked all participants to evaluate all the 24 images from our laboratory replication studies. We sought to obtain a fine-grained measure of image evaluations asking the participants to evaluate the images on five dimensions. Thus, for each image we asked participants to state if they disagreed or agreed with five statements about their emotional reactions to the images: "I have a strong emotional reaction" (Emotion Strength), "I feel uncomfortable" (Uncomfortable), "I feel happy" (Happy), "I feel threatened" (Threatened), and "I feel disgusted" (Disgusted). We standardized all variables to have mean 0 and standard deviation 1.11

11 The survey also included two measures of political ideology: a 5-item Social Conservatism scale $(\alpha=.83)$ and a 5-item Economic Conservatism scale $(\alpha=.80)$. In Online Appendix 3D, we replicate the analyses from the previous test and find that in Denmark self-reported perceptions of threat in images is a significant positive predictor of social but not economic conservatism and the difference in these associations is itself significant. 
To test whether the link between physiological reactions and political ideology depends on the underlying characteristics of the images, we combined our laboratory data on physiological readings and political ideology with the two types of ratings of the different images from our survey sample. To this end, we estimated a series of models where we regressed our measures of political ideology on physiological reactions to the images, the ratings of the images on the separate dimensions, as well as the interactions between the two variables. To make maximal use of the available data, our key units of analysis are responses to a specific image. Because each of our participants had multiple responses, we cluster the standard errors by subject to correct for within-participant autocorrelation. In addition, we control for the same set of covariates as in the previous tests.

\section{Results}

We present the key statistical analyses in Online Appendix 4A-B and summarize the findings here. Turning first to the analyses where we focus on participants' own self-reported image ratings, we find no evidence of an interaction effect. In neither Denmark nor the United States does the association between EDA responses to the images and political ideology depend on self-reported valence or arousal ratings of the images (see Table 4A.a-d and Figure 4A.a-b in the Online Appendix). Thus, even when we take into account the fine-grained properties of the images (i.e., self-reported valence and arousal ratings), we fail to obtain a relationship between physiological reactions and ideology. When we turn to the analyses with the even more detailed survey ratings of the images - i.e., where survey

participants rated the images on five dimensions - we obtain essentially similar results. We find occasional hints that the association between EDA responses and political ideology is stronger among more negatively rated images in the United States (see Figure 4B.1 in the Online Appendix). But in Denmark, and when we examine the combined Danish and US samples, the interaction effects between physiological responses and self-reported ratings are insignificant. And when we examine the threeway EDA Response X Self-Reported Rating X Country interactions, we find that the differences 
between the results from Denmark and the United States are not statistically different from each other (all $p$ 's $>.14)$.

The hope was that an integration, on the one hand, data about (1) self-reported reactions to pictures and (2) the properties of individual pictures with, on the other hand, data about the physiological reactions to these pictures would increase the ability to detect associations between political ideology and physiological reactions. Overall, the inconclusive nature of the findings suggest that the reliability issues identified in the replication studies are severe and cannot in any simple way be counter-balanced through an increase in the measurement validity of the utilized measures.

\section{A Meta-Analysis of All Published Studies}

Based on the above conclusions, an important question is the extent to which the identified issues of reliability and validity are study- or method-specific. In other words, are these issues specific to the present study or do they also plague previous studies? To asses this, our final test is a meta-analysis of all published studies that allow us to assess an association between physiological measures of threatsensitivity and political ideology, examining not only these associations but also the properties of the utilized physiological measures.

\section{Sampling}

As discussed in Online Appendix Section 1, we identified seven existing studies of lay people that included measures of EDA responses to negative images and political ideology: Aarøe et al. (2017), Coe et al. (2017), Dodd et al. (2012), Knoll et al. (2015), Oxley et al. (2008), Petersen et al. (2015) and Smith et al. (2012). Table 1 provides an overview of the studies, including their country location, sample size, types of images included (and those used to generate their measure of threat-sensitivity), the specific method for analyzing the physiological data, the included ideological measures, as well as our tests of the reliability and validity of the measures. In assessing the totality of the evidence, it is 
crucial to note that Oxley et al. (2008) and Dodd et al. (2012) are based on the exact same underlying data but differ in terms of analytical choices (i.e., the images and political ideological measures they chose to include in the analyses and the way they calculated physiological reactions to the images). Also, as noted in Smith et al. (2012), 9 individuals from Oxley et al. (2008) were also invited to participate in this study (equaling $18 \%$ of the total sample).

To obtain physiological measures, all studies follow a template similar to Oxley et al. (2008), but they differ in their specific methods for estimating changes in EDA in response to images. This will turn out to be important. Knoll et al. (2015), Oxley et al. (2008), Smith et al. (2012), and Coe et al. (2017) used the same "log-and-subtract" method as described above. Dodd et al. (2012) use a similar approach but index the proportion rather than the difference. To facilitate comparison, we have here recoded their data to follow the original setup of Oxley et al. (2008). Finally, Aarøe et al. (2017) and Petersen et al. (2012) followed a recent recommendation (Figner \& Murphy 2011: 167) and calculate the area bounded by the phasic curve, measured between 1 second after stimulus onset to stimuli offset. Because this approach does not rely on ISIs to correct for baseline variations in EDA, the datasets from these studies did not include ISI measures.

\section{Assessment of Reliability and Measurement Validity}

To examine the reliability of the physiological measures used in existing studies, we first examine whether participants' EDA responses to threatening and disgusting images correlated with their EDA responses from the preceding inter-stimulus intervals. Because Aarøe et al. (2017) and Petersen et al. (2012) used the area bounded by a curve and hence did not include data on ISIs, it is impossible to carry out this specific test for these two studies. The tests reported in Table 1 demonstrate that participants' reactions to negative images largely resemble their baseline responsiveness. In all five studies, participants' reactions to negative images closely mirrored their reactions during the previous inter-stimulus interval, all r's $>.99$. Thus, the signal captured by the difference score is at best very 
weak. As noted previously, this is especially problematic for EDA measures where the risk of a low signal-to-noise ratio is already high, given the many potential confounding factors. Second, we calculate Cronbach's $\alpha$ for scales consisting of changes in EDA during exposure to the various threatening images for each of the seven studies. As displayed in Table 1 , the $\alpha$-coefficients range from .04 in Dodd et al. (2012) to .74 in Petersen et al. (2017). While Dodd et al. (2012) and Oxley et al. (2008) rely on the same participants and have in common two of the three images, the $\alpha$-coefficient in Dodd et al. (2012) is much lower than in Oxley et al. (2008) because their last image (i.e., a man fighting a crowd) correlates negatively with the two others. Finally, an interesting observation is that the two studies using "the area-bounded-by-the-curve" method have markedly higher reliability (Aarøe et al. 2017; Petersen et al. 2015).

To speak to convergent validity, we examined the average inter-item correlations for reactions to individual threatening images. Again, these vary considerably from one study to another, ranging from .04 in Knoll et al. (2015) to .58 in Petersen et al. (2015). Again, we observe that the two studies utilizing the "the area-bounded-by-the-curve" method have markedly higher correlations.

Finally, to assess discriminant validity, we examine the average inter-item correlations between reactions to images of different types. In Oxley et al. (2008) and Dodd et al. (2012), we compare negative images to the same three positive images. In Aarøe et al (2017) we compare reactions to negative images to reactions to both positive and neutral images. Finally, in the Petersen et al. (2015) and Knoll et al. (2015) studies, we compare reactions to negative images to reactions to non-negative images, i.e., a mix of both positive and neutral images. Coe et al. (2017) and Smith et al. (2012) only provided information on negative images, and we therefore cannot carry out this validity test for their studies. The first main finding to emerge is that in the studies that rely on the "log-and-subtract" method, the correlations between negative images and other image types are very low: ranging from .10 in Oxley et al. (2015) to -.01 in Knoll et al. (2012). This suggest that people react differently to negative images than to other types of images. The second main finding is that EDA responses to 
negative images correlate highly with reactions to other image types in Aarøe et al. (2017) and Petersen et al. (2015), the two studies that rely on the "area-bounded-by-the-curve" approach. Thus, while these studies have satisfactory convergent validity, they do not appear to have high degrees of discriminant validity.

Overall, this assessment of the published literature suggests the reliability and validity issues identified in the replications are method-specific rather than something produced in the present replications. In essence, studies using versions of the "log-and-subtract" method have not relied on measures with satisfactory degrees of reliability or measurement validity. This naturally limits the weight of the empirical evidence provided by these studies on the existence of an association between physiological measures of threat-sensitivity and political ideology. Studies using the "area-boundedby-the-curve" method fare better but their failure to discriminate between physiological reactions to threatening and positive images suggests that these measures are essentially indicators of individual differences in arousal and physiological reactivity rather than individual differences in sensitivity to particular valences, like threat. This is exactly what EDA has been validated in the psychophysiological literature to reliably measure (Lang et al., 1993; Frith \& Allen, 1983). At the same time, it suggests that efforts to measure threat sensitivity specifically using EDA will be fraught with difficulty. This is a key issue and we return to it in the discussion. 
Table 1. Overview and measurement properties of published studies with ideological and skin conductance measures.

\begin{tabular}{|c|c|c|c|c|c|c|c|c|}
\hline Study & Country & $\mathbf{N}$ & $\begin{array}{c}\text { Image Types } \\
(* \text { images used for } \\
\text { threat-sensitivity) }\end{array}$ & Method & $\begin{array}{l}\text { Ideology } \\
\text { Measures }\end{array}$ & $\begin{array}{l}\text { Correlation: } \\
\text { Image and ISI }\end{array}$ & $\begin{array}{l}\alpha \text { and Average Inter- } \\
\text { item Correlation }\end{array}$ & $\begin{array}{l}\text { Correlation: } \\
\text { Negative } \\
\text { Images and } \\
\text { Other Image } \\
\text { Types }\end{array}$ \\
\hline Aarøe et al. & Denmark & 42 & $\begin{array}{l}6 \text { highly disgusting; } \\
8 \text { mildly disgusting; } \\
10 \text { positive; } 4 \text { neutral }\end{array}$ & $\begin{array}{l}\text { Area-under- } \\
\text { the-Curve }\end{array}$ & $\begin{array}{l}\text { Left-Right Self-placement } \\
\text { Social Conservatism }\end{array}$ & NA & $\begin{array}{l}\alpha \mathrm{High} \text { Disgust } 2=.71 \\
\text { CorrHigh Disgust }=.29\end{array}$ & $\begin{array}{l}\text { CorrNegative }- \\
\text { Positive }=.86 \\
\text { CorrNegative }- \\
\text { Neutral }=.75\end{array}$ \\
\hline Oxley et al. & $\begin{array}{l}\text { United } \\
\text { States }\end{array}$ & 46 & $\begin{array}{l}3 \text { threatening; } * 3 \\
\text { positive }\end{array}$ & $\begin{array}{l}\text { Log-and- } \\
\text { Subtract }\end{array}$ & Social Conservatism & CorrThreat $=.996$ & $\begin{array}{l}\alpha \text { Threat }=.29 \\
\text { CorrThreat }=.12\end{array}$ & $\begin{array}{l}\text { CorrNegative }- \\
\text { Positive }=-.10\end{array}$ \\
\hline Dodd et al. & $\begin{array}{l}\text { United } \\
\text { States }\end{array}$ & 46 & $\begin{array}{l}3 \text { threatening; } * 3 \\
\text { positive }\end{array}$ & $\begin{array}{l}\text { Log-and- } \\
\text { Subtract } 1\end{array}$ & $\begin{array}{l}\text { Social Conservatism } \\
\text { Left-Right self-placement }\end{array}$ & CorrThreat $=.995$ & $\begin{array}{l}\alpha \text { Threat }=.04 \\
\text { CorrThreat }=.01\end{array}$ & $\begin{array}{l}\text { CorrNegative }- \\
\text { Positive }=-.04\end{array}$ \\
\hline Smith et al. & $\begin{array}{l}\text { United } \\
\text { States }\end{array}$ & 50 & 3 disgusting* & $\begin{array}{l}\text { Log-and- } \\
\text { Subtract }\end{array}$ & $\begin{array}{l}\text { Social Conservatism } \\
\text { Left-Right self-placement }\end{array}$ & CorrThreat $=.996$ & $\begin{array}{l}\alpha \text { Threat }=.40 \\
\text { CorrThreat }=.18\end{array}$ & NA \\
\hline $\begin{array}{l}\text { Petersen et } \\
\text { al. }\end{array}$ & Denmark & 58 & $\begin{array}{l}2 \text { negative; } * 6 \\
\text { positive/neutral }\end{array}$ & $\begin{array}{l}\text { Area-under- } \\
\text { the-Curve }\end{array}$ & $\begin{array}{l}\text { Left-Right Self-Placement } \\
\text { Social Conservatism } \\
\text { Economic Conservatism }\end{array}$ & NA & $\begin{array}{l}\alpha \text { Threat }=.74 \\
\text { CorrThreat }=.58\end{array}$ & $\begin{array}{l}\text { CorrNegative }- \text { Non- } \\
\text { Negative }=.72\end{array}$ \\
\hline Coe et al. & $\begin{array}{l}\text { United } \\
\text { States }\end{array}$ & 182 & 6 threatening* & $\begin{array}{l}\text { Log-and- } \\
\text { Subtract }\end{array}$ & Left-Right Self-Placement & CorrThreat $=.996$ & $\begin{array}{l}\alpha \text { Threat }=.57 \\
\text { CorrThreat }=.18\end{array}$ & NA \\
\hline Knoll et al. & $\begin{array}{l}\text { United } \\
\text { States }\end{array}$ & 60 & $\begin{array}{l}6 \text { threatening; } * 3 \\
\text { non-threatening }\end{array}$ & $\begin{array}{l}\text { Log-and- } \\
\text { Subtract }\end{array}$ & $\begin{array}{l}\text { Social Conservatism } \\
\text { Economic conservatism }\end{array}$ & CorrThreat $=.984$ & $\begin{array}{l}\alpha \text { Threat }=.20 \\
\text { CorrThreat }=.04\end{array}$ & $\begin{array}{l}\text { CorrNegative }- \text { Non- } \\
\text { Negative }=-.01\end{array}$ \\
\hline
\end{tabular}

Note. See Online Appendix Section A for details on sampling.1The original study uses ratio (stimulus/inter-stimulus) instead of log-and-subtract; 2 Before calculating alpha and average inter-item correlations, we standardized the items to account for differences in standard deviations. 
Results

Setting aside these non-trivial measurement concerns, we use OLS regression to estimate the relationship between the measure of threat-sensitivity and political ideology for each of the seven studies separately and for each available ideology measure. To compare the strength of association across studies, we standardized our physiological and ideological measures to have a mean of 0 and a standard deviation of 1. Finally, we note that Oxley et al.'s (2008) preferred model includes controls for income, education, age and gender. While not all of the data we obtained from the studies in the meta-analysis include those same variables, for each study, we include as many from the list as possible. Results are presented in Figure 3.

Figure 3. Associations between four measures of political ideology and EDA responses to negative images in seven published studies.

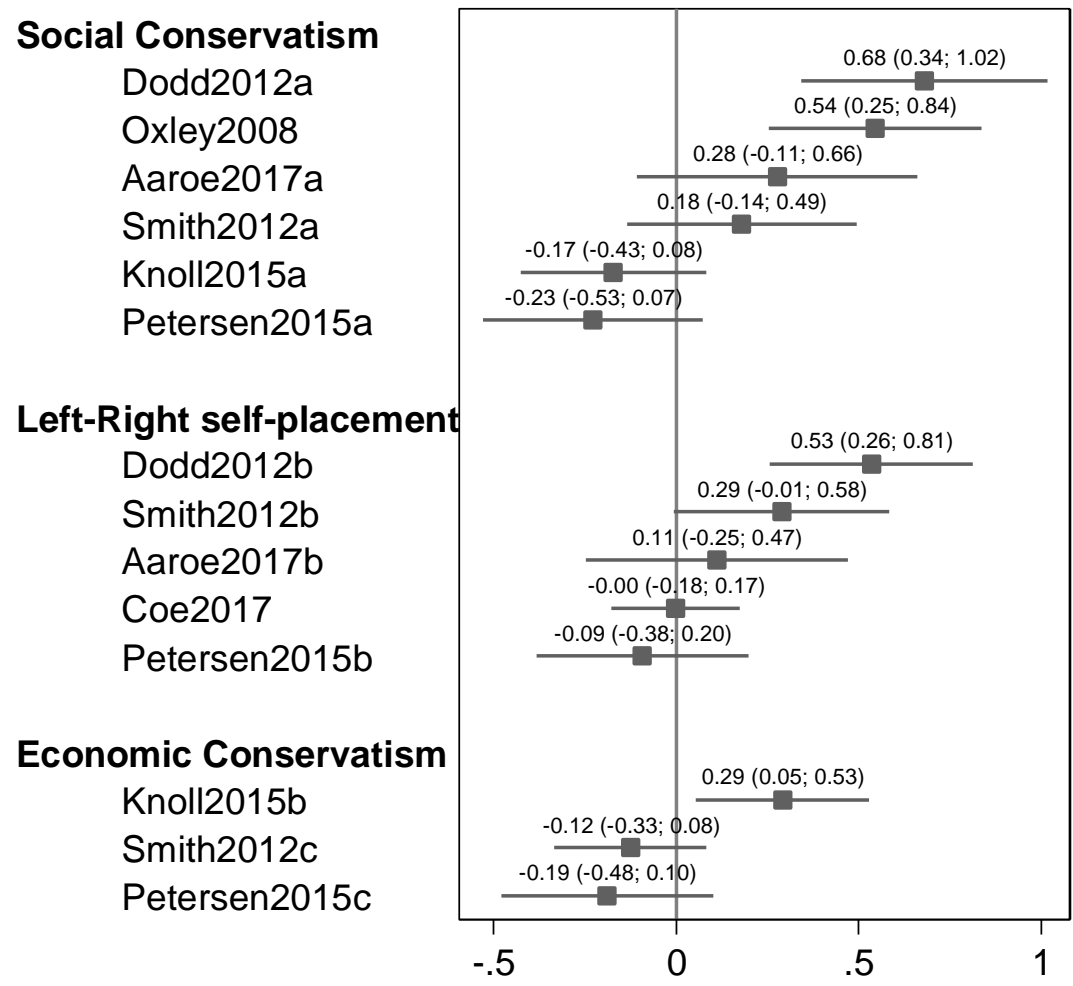

Notes. Entries are coefficient estimates from OLS regression analyses of the associations between the ideological EDA response measures. All variables scored with a mean of 0 and a standard deviation of 1 . For study details, see Table 1 . Note that participants in Dodd et al. (2012) and Oxley et al. (2008) are identical, just as the studies rely on partially the same images and ideological measures. 
First, we find a positive association between EDA responses to negative images and right-wing ideology in seven out of 14 tests, but only five of these are statistically significant at the .05-level (i.e., Dodd 2012a, Oxley 2008, Dodd 2012b, Smith 2012b, Knoll 2015b in Figure 3). In contrast, we find a negative relationship between EDA responses and conservatism in six tests, but none of these are statistically distinguishable from zero. Second, the findings are similar across our three measures of political ideology in that the different studies contain both negative and positive associations, though the relationship between EDA and social conservatism shows the most consistent pattern: Here four out of six coefficient estimates are positive, although it is important to emphasize again that the two significant estimates (Dodd 2012a, Oxley 2008) come from studies that rely on the same samples and partially the same images. Third, the estimated coefficients vary considerably, from -.23 in Petersen et al. (2015) to .68 in Dodd et al. (2012).

It is relevant to observe that neither the unreliable measures of individual differences in threat-sensitivity nor the more reliable measures of individual differences in arousal (i.e., from Petersen et al. and Aarøe et al. using the "area-bounded-by-the-curve"-approach) show consistent associations with ideology. Hence, even without considering the reliability and validity issues, the available data do not produce consistent evidence for conservatives being either higher in threatsensitivity nor higher in arousal as measured by EDA. (Because many of the associations in Figure 1 come from the same studies and are correlated with one another, we opted to not combine the results to produce an "overall meta-analytical effect" (e.g., Harbord and Higgins, 2008) across the different tests.)

To examine these claims further, we recoded the EDA measures from our own replication studies following the "area-bounded-by-the-curve" approach. Consistent with the findings from the meta-analysis, we also find that this method yields reliable measures ( $\alpha$ threat images, United States $=.90 ; \alpha$ 
threat images, Denmark $=.84) .12$ We then reexamine the main effects of these measures on political ideology as well as the interaction effects with the ratings of the images. We present the analyses in Online Appendix 5A. The analyses provide little evidence that these reliable measures are consistently related to political ideology, whether economic or social in nature, or whether in the United States or Denmark. Furthermore, we conducted additional analyses to assess which particular feature of the "areabounded-by-the-curve" approach yielded the increase in reliability. We show the analyses in the Online Appendix, section 5A.1. Briefly, the results show that it is specifically the transformation of the EDA signal from a tonic signal to a phasic signal, which involves the removal of between-participant differences in baseline EDA levels and within-participant drift in the EDA signal by imposing a socalled high pass filter (Murphy \& Figner, 2012).

\section{Conclusion}

In this article, we have undertaken the most thorough assessment to date of the association between individual differences in skin conductance reactions to negative images and individual differences in political ideology. The existence of such an association has emerged as a key finding in recent research on political behavior and has paved the way for the increased use of physiological measures outside the study of political ideology. Consequently, it is important to pause and take stock of current methods and findings.

Our focus has been methodological rather than theoretical. The theory that self-reported experiences of threat, uncertainty and negativity are associated with political conservatism has been subject to repeated tests over several decades using a large number of different methods and is, in our view, well-supported. Hence, our research question is whether studies using psychophysiological measures designed to capture EDA when processing threatening pictures are reliably able to reproduce

12 In the Appendix, Table 5A.c-d, we show that our area-bounded-by-the-curve measures do not correlate strongly with the log-and-subtract-measures presented earlier in the manuscript, nor do they correlate with self-reported reactions to the images. 
this association. Specifically, we asked about the replicability, reliability, measurement validity and external validity of this association, when assessed using EDA.

By combining all available published data on psychophysiological reactions and political ideology among lay individuals with two novel, large-scale replication studies, we found limited replicability of the ideology-physiology link. The association between heightened physiological reactions to negative or threatening images and conservatism has been identified in three existing analyses of samples from Nebraska (if we include the Dodd et al. (2012) paper that relies on the same participants as the original study by Oxley et al. [2008]). We were able to replicate this association in our own sample collected from the same population. However, other existing studies from other US locations and Denmark could not identify the association, nor were we able to replicate it with our new Danish replication study. This suggests that the association has limited external validity. Importantly, tests using self-reported emotional reactions to images suggested that this does not reflect a lack of association between the strength of reactions to threatening pictures and political ideology outside the context of the original study by Oxley et al. (2008). In both the Danish and US replication samples, we found consistent and significant associations in the expected direction using these self-reported measures.

In our analyses, we have consistently compared potentially distinct reactions in the form of feelings of disgust and feelings of threat. The measurement properties of the physiological measures make it difficult to draw any strong inferences but if we include the findings using self-reported measures, the totality of the evidence suggests that if there is an association between political ideology and reactions to images, then this association is more reliably related to feelings of threat than feelings of disgust. We have also consistently compared associations for economic and social conservatism. With the same caveats in mind, there is some evidence from the self-reported reactions for stronger associations between threat-sensitivity and social conservatism compared to economic conservatism. 
Overall, our analyses suggest that the fickle nature of the association between psychophysiological measures of threat-sensitivity and political ideology reflects poor reliability and measurement validity. Proponents of the most widespread extraction method, log-and-subtract, argue it allows researchers to examine EDA responses to specific stimuli (e.g., threatening images). But data from our re-analyses of existing studies and two replication studies suggest that estimates from the log-and-subtract method are extremely noisy and fall far below conventional standards of reliability and convergent validity. An alternative method, the area-bound-by-the-curve method based on the phasic EDA signal, yielded reliable measures. Yet, these measures do not seem to track individual differences in threat sensitivity. Instead, they seem to track individual differences in general psychophysiological reactivity. This is consistent with recent studies in psychophysiology. For example, Bulteel et al. (2014: 39) conclude, "there is large idiographic variation in individuals' physiological responses to emotional events, and hence, in the nature of response patterning that will be observed in different individuals." In other words, the individual differences in general physiological responsiveness are so large that it becomes extremely difficult to capture individuallevel variation in responsiveness to particular types of stimuli, like threatening images. In this way, the identified issues have less to do with psychophysiological measures themselves and more to do with the use of these measures within political science. In essence, electrodermal activity has been utilized to measure something that they cannot, in fact, measure.

There is general agreement that stronger skin conductance responses to images track how arousing these images are (Lang et al., 1993). It therefore seems natural if the phasic (and reliable) individual difference measure of general physiological responsiveness tracks individual differences in arousal. If valid, the lack of correlation between the phasic measure and measures of political ideology in both United States and Denmark becomes relevant for current debates in political psychology. Thus, Tritt et al. (2014) suggest that individual differences in arousal are related to political ideology such 
that conservatives are more easily aroused. The present analyses, however, provide little supporting evidence for this claim.

At the same time, the relationship between EDA and the "somewhat vague entity arousal" is not straightforward (Frith \& Allen, 1984: 35). Thus, Frith \& Allen (1984) conclude that EDA does not simply track emotional arousal but rather "short term changes" (p. 38) in "the general engagement of attention during performance of any task" (p. 35), which could occur due to changes in arousal. Consistent with this, the phasic measures of skin conductance reactivity are highly associated across image types including neutral (and, presumably, non-arousing) images.13 Thus, one possible interpretation is that the phasic, reliable individual difference measure extracted in the present analyses is a measure of individual differences in psychophysiological reactivity associated with attentional shifts. In the context of the present studies, these shifts occurred when attention was turned towards an image following a blank screen. In support of this, difference-of-means t-tests reveal that phasic physiological reactions are stronger during exposure to the images than during the inter-stimulus intervals in both our Danish $(\mathrm{t}=9,35, \mathrm{p}<.001)$ and $\mathrm{US}(\mathrm{t}=7,75, \mathrm{p}<.001)$ replications.

All in all, the present findings demonstrate beyond any reasonable doubt that political scientists should show extraordinary care when collecting, analyzing and interpreting psychophysiological measures. Furthermore, the present analyses provide a clear set of recommendations for researchers committed to such an endeavor. On the basis of the present findings, we first recommend that political scientists always subject psychophysiological measures to standard tests of reliability and measurement validity and that any findings be interpreted in light of the measurement properties of the measurement. Second, we recommend that political scientists always collect alternative measures with higher levels of measurement reliability and validity and validate the conclusions from psychophysiological measures with these more robust measures, including self-

13 See also Online Appendix 5A that documents that EDA responses are not associated with particular types of ratings of pictures. 
reports. Third, we recommend that political science researchers take significant steps to improve the measurement properties of physiological measures. One way ahead is to more closely mirror the protocols used when electrodermal activity is deployed as a diagnosis tool in clinical studies. This includes, e.g., periods of relaxation before data acquisition and a minimization of external influences such as noises (see also Murphy \& Figner, 2011; Tomarken, 1995). But given that widespread approaches like the log-and-subtract method yields extremely noisy measures, it also means that political scientists should consider alternatives. On the basis of the present findings, we echo Murphy \& Figner (2011) and recommend that researchers instead use the area-under-the-curve approach, or other approaches relying on the phasic signal, for extracting the measures.

As we have suggested, the phasic measures do not seem to capture individual differences in threat-sensitivity but more general individual differences in the psychophysiological correlates of attentional shifts. Our most basic recommendation is to integrate this directly into the studies of the political correlates of psychophysiological individual differences. Rather than try to measure something that cannot be reliably measured with psychophysiology equipment, political scientists interested in psychophysiology should build hypotheses directly from theories of the type of psychophysiological activity that can in fact be reliably captured. Gruszczynski et al. (2013), for example, show that individuals with higher electrodermal reactivity tend to participate more in politics. It is indeed plausible that people who react more at the physiological level to contextual shifts are more drawn to the hustle and bustle of politics. Further studies along such lines could hold more promise for the use of psychophysiology in political science research.

\section{Acknowledgements}

We thank Lene Aarøe and John Hibbing for valuable help with the design and collection of the replication studies. For valuable advice and comments in the preparation of the manuscript, we thank Kevin Arceneaux, Bert Bakker, Andrew Gelman, Frank Gonzales, Dan Mønster, John Peterson and 
Gijs Schumacher. For insights on the difference between the different extraction methods, we are, in particular, indebted to Stuart Soroka. Finally, we thank all authors of the analyzed studies for generously sharing their data with us. Previous versions of this manuscript were presented at the 2016 annual meetings of the American Political Science Association and the Danish Political Science Association and at the 2017 annual meeting of Midwest Political Science Association.

\section{References}

Aarøe, L., Petersen, M. B., \& Arceneaux, K. (2017). The behavioral immune system shapes political intuitions: Why and how individual differences in disgust sensitivity underlie opposition to immigration. American Political Science Review, 111(2), 277-294.

Bert N. Bakker, B., Schumacher, G., Gothreau, C., \& Arceneaux, K. (2019). Conservatives and Liberals have Similar Physiological Responses to Threats. Evidence from Three Replications. Preprint: https://osf.io/js9r5/.

Bulteel, K., Ceulemans, E., Thompson, R. J., Waugh, C. E., Gotlib, I. H., Tuerlinckx, F., \& Kuppens, P. (2014). DeCon: A tool to detect emotional concordance in multivariate time series data of emotional responding. Biological Psychology, 98, 29-42.

Carney, D. R., Jost, J. T., Gosling, S. D., \& Potter, J. (2008). The secret lives of liberals and conservatives: Personality profiles, interaction styles, and the things they leave behind. Political Psychology, 29(6), 807-840.

Coe, C. M., Canelo, K. S., Vue, K., Hibbing, M. V., \& Nicholson, S. P. (2017). The physiology of framing effects: Threat sensitivity and the persuasiveness of political arguments. The Journal of Politics, 79(4), 1465-1468.

Dodd, M. D., Balzer, A., Jacobs, C. M., Gruszczynski, M. W., Smith, K. B., \& Hibbing, J. R. (2012). The political left rolls with the good and the political right confronts the bad: connecting 
physiology and cognition to preferences. Philosophical Transactions of the Royal Society B: Biological Sciences, 367(1589), 640-649.

Figner, B., \& Murphy, R. O. (2011). Using skin conductance in judgment and decision making research. A handbook of process tracing methods for decision research, 163-184.

Frith, C. D., \& Allen, H. A. (1983). The skin conductance orienting response as an index of attention. Biological psychology, 17(1), 27-39.

Gerber, A. S., Huber, G. A., Doherty, D., Dowling, C. M., \& Ha, S. E. (2010). Personality and political attitudes: Relationships across issue domains and political contexts. American Political Science Review, 104(1), 111-133.

Gruszczynski, M. W., Balzer, A., Jacobs, C. M., Smith, K. B., \& Hibbing, J. R. (2013). The physiology of political participation. Political Behavior, 35(1), 135-152.

Harbord, R. M., \& Higgins, J. P. (2008). Meta-regression in Stata. The Stata Journal, 8(4), 493-519.

Hatemi, P. K., \& McDermott, R. (2012). The genetics of politics: Discovery, challenges, and progress. Trends in Genetics, 28(10), 525-533.

Hibbing, J. R. (2013). Ten misconceptions concerning neurobiology and politics. Perspectives on Politics, 11(2), 475-489.

Hibbing, J. R., Smith, K. B., \& Alford, J. R. (2014). Differences in negativity bias underlie variations in political ideology. Behavioral and Brain Sciences, 37(3), 297-307.

Jost, J. T. (2006). The end of the end of ideology. American psychologist, 61(7), 651.

Jost, J. T., \& Amodio, D. M. (2012). Political ideology as motivated social cognition: Behavioral and neuroscientific evidence. Motivation and Emotion, 36(1), 55-64.

Jost, J. T., Banaji, M. R., \& Nosek, B. A. (2004). A decade of system justification theory: Accumulated evidence of conscious and unconscious bolstering of the status quo. Political psychology, 25(6), 881-919. 
Jost, J. T., Federico, C. M., \& Napier, J. L. (2009). Political ideology: Its structure, functions, and elective affinities. Annual review of psychology, 60, 307-337.

Knoll, B. R., O’Daniel, T. J., \& Cusato, B. (2015). Physiological responses and political behavior: Three reproductions using a novel dataset. Research \& Politics, 2(4), 2053168015621328.

Lang, P. J., Greenwald, M. K., Bradley, M. M., \& Hamm, A. O. (1993). Looking at pictures: Affective, facial, visceral, and behavioral reactions. Psychophysiology, 30(3), 261-273. Malka, A., Lelkes, Y., \& Soto, C. J. (2017). Are cultural and economic conservatism positively correlated? A large-scale cross-national test. British Journal of Political Science, 1-25.

Manuck, S. B., Kasprowicz, A. L., Monroe, S. M., Larkin, K. T., \& Kaplan, J. R. (1989). Psychophysiologic reactivity as a dimension of individual differences. In Handbook of research methods in cardiovascular behavioral medicine (pp. 365-382). Springer, Boston, MA.

Merolla, J. L., \& Zechmeister, E. J. (2009). Democracy at risk: How terrorist threats affect the public. University of Chicago Press.

Ogorevc, J., Geršak, G., Novak, D., \& Drnovšek, J. (2013). Metrological evaluation of skin conductance measurements. Measurement, 46(9), 2993-3001.

Open Science Collaboration. (2015). Estimating the reproducibility of psychological science. Science, 349(6251), aac4716.

Oxley, D. R., Smith, K. B., Alford, J. R., Hibbing, M. V., Miller, J. L., Scalora, M., ... \& Hibbing, J. R. (2008). Political attitudes vary with physiological traits. science, 321(5896), 1667-1670.

Petersen, M. B., Giessing, A., \& Nielsen, J. (2015). Physiological responses and partisan bias: Beyond self-reported measures of party identification. PloS one, 10(5), e0126922.

Peterson, J. C., Smith, K. B., \& Hibbing, J. R. (2016). Physiology and political beliefs: A response to Knoll, O’Daniel, and Cusato. Research \& Politics, 3(3), 2053168016662892.

Slothuus, R., Stubager, R., Hansen, K. M., Petersen, M. B., \& Pettersson, M. (2010). Måling af politiske vardier og informationsbearbejdning. Nye indeks for fordelingspolitik, vardipolitik og 
"Need to Evaluate" blandt danske veelgere. Department of Political Science and Government, University of Aarhus, Denmark.

Smith, K. B., Oxley, D., Hibbing, M. V., Alford, J. R., \& Hibbing, J. R. (2011). Disgust sensitivity and the neurophysiology of left-right political orientations. PloS one, 6(10), e25552.

Soroka, S. N. (2019). Skin Conductance in the Study of Politics and Communication. In Biophysical Measurement in Experimental Social Science Research (pp. 85-104). Academic Press.

Terrizzi Jr, J. A., Shook, N. J., \& McDaniel, M. A. (2013). The behavioral immune system and social conservatism: A meta-analysis. Evolution and Human Behavior, 34(2), 99-108.

Tomarken, A. J. (1995). A psychometric perspective on psychophysiological measures. Psychological Assessment, 7(3), 387.

Tritt, S. M., Inzlicht, M., \& Peterson, J. B. (2014). Confounding valence and arousal: What really underlies political orientation?. Behavioral and Brain Sciences, 37(3), 330-331. 


\section{Biographical Statements}

Mathias Osmundsen is an Assistant Professor at Aarhus University, Aarhus, DK-8000 Aarhus C.

David J. Hendry is an Assistant Professor at London School of Economics, London, WC2A 2AE.

Lasse Laustsen is an Associate Professor at Aarhus University, Aarhus, DK-8000 Aarhus C. Kevin

B. Smith is a Professor at University of Nebraska, Lincoln, NE 68588-0328. Michael Bang Petersen is a Professor at Aarhus University, Aarhus, DK-8000 Aarhus C. 